\title{
Estudo Multicêntrico do Implante de Stent Intracoronário no Rio de Janeiro
}

\author{
N orival Romão, Esmeralci Ferreira, Cláudio A. Feldman, Renato Vilella, N oé Costa, Cyro V. Rodrigues, \\ A ndré Pessanha, Valério Fuks, Sérgio M. Leandro
}

Rio de Janeiro, RJ

Objetivo - Avaliar retrospectivamente, implante de stent (IS) em paciente com doença arterial coronária (DAC), realizado em 7 hospitais do Rio de Janeiro.

Métodos - De junho/94 a dezembro/96, foram realizados 2.220 procedimentos, e desses, analisados 783 IS em 660 (29,7\%) pacientes, através da coronariografia sem subtração digital. Os diversos tipos de stent, mais freqüentemente, usados foram: Palmaz-Schatz (40,9\%), Gianturco-Roubin $(29,1 \%)$ e NIR (22,0\%). As indicações para o IS foram: lesão de novo, 67,9\%; lesão reestenótica, 16,0\%; lesão com resultado subótimo da angioplastia transluminal coronária (ATC), 8,2\%; oclusão aguda ou provável pós-ATC, 4,9\%; oclusão crônica, 3,0\%. Todos os stents foram implantados com alta pressão, sem controle pelo ultra-som intracoronário. A prevenção da trombose subaguda, na maioria dos pacientes $(87,8 \%)$, foi feita com o uso de ticlopidina e ácido acetil-salicílico.

Resultados - Evolução imediata: a) sucesso no IS em 770 lesões: $98,0 \%$ em $646(97,9 \%)$ pacientes; $b$ ) sucesso clínico em 634 (96,0\%) casos; c) complicações maiores: infarto agudo do miocárdio (IAM) - 1,1\%; cirurgia de revascularização miocárdica (CRM) - 1,4\% e óbito $0,8 \%$; d) complicações vasculares com correção cirúrgica elou sangramento - 3,0\%. Evolução tardia: a) dos 399 $(60,4 \%)$ pacientes acompanhados clinicamente, a coronariografia de 121 (30,3\%) mostrou retorno da lesão no IS em $79(19,8 \%)$ casos; b) freqüência de outros eventos: IAM - 1,5\%; CRM - 2,3\%; óbito -1,0\% e outra ATC ou procedimento similar $-12,5 \%$.

Conclusão - O estudo multicêntrico mostrou que o IS na DAC pode ser realizado com segurança, alto índice de sucesso imediato, poucas complicações e baixa taxa de eventos cardíacos na evolução tardia.

Palavras-chave: implante de stent, doença arterial coronária, reestenose coronária

\section{Multicenter Study of Intracoronary Stent Implantation in Rio de Janeiro}

Purpose - To evaluate retrospectively stent implantation (SI) in patients with coronary artery disease $(C A D)$ performed in 7 hospitals in Rio de Janeiro.

Methods - From June/94 to December/96 2,220 procedures were performed among which we analyzed 783 SI in $660(29.7 \%)$ patients using coronary angiography without digital subtraction. Several types of stents were used: Palmaz-Schatz(40.9\%), Gianturco-Roubin (29.1\%) e NIR (22.0\%). Indications for SI: 1 - de novo lesion (67.9\%); 2) restenotic lesion (16.0\%); 3) sub-optimal results after PTCA (8.2\%); 4) abrupt or threatened closure after PTCA (4.9\%); 5) chronic occlusion (3.0\%). All stents were implanted using high pressure balloon inflation without intracoronary ultrasound guidance. Sub-acute stent thrombosis prevention, in the majority of patients (87.8\%) was done with ticlopidine and aspirin.

Results - Early outcome: a) the success rate of SI in 770 lesions was $98.0 \%$ in 646 (97.9\%) patients; $b$ ) the clinical success rate in 634 patients was $96.0 \%$; c) the major complications were acute myocardial infarction (1.1\%); coronary artery bypass graft (1.4\%) and death $(0.8 \%) ; d)$ vascular complications with surgical correction and/or bleeding occurred in 3.0\%. Late outcome: a) the clinical follow-up of $399(60.4 \%)$ and the coronary angiographies of 121 patients (30.3\%) showed in-stent lesion in $79(19.8 \%)$; b) other event rates: myocardial infarction (1.5\%); coronary bypass (2.3\%); death: $1.0 \%$ and other PTCA or similar procedure (12.5\%).

Conclusion - This multicentric study showed that SI for CAD can be performed with safety, high early success rate, few complications and low rate of cardiac events during the late follow-up.

Key-words: stents implantation, coronary artery disease, coronary restenosis

Arq Bras Cardiol, volume $70\left(n^{0} 1\right), 37-42,1998$

Hospitais: Prontocor (Prontocine Serviço de Hemodinâmica), São Lucas, São Vicente de Paula, Rio Mar, Cardiologia Laranjeiras, Adventista Silvestre, Sincordis - Rio de Janeiro

Correspondência: Norival Romão - Av. Sernambetiba, 3300/507 - Bl. 6 - 22630010 - Rio de Janeiro, RJ

Recebido para publicação em 29/8/97

Aceito em 30/10/97
Embora Dotter ${ }^{1}$ tivesse sugerido, em 1969, que o implante de stent (IS) pudesse auxiliar no tratamento da estenose vascular, somente após duas décadas, a experiência com stent para prevenir a oclusão aguda e a reestenose foi utilizada por Sigwart ${ }^{2}$ nos pacientes submetidos a angioplastia transluminal coronária (ATC). Atualmente, o 
IS é uma técnica estabelecida, que diminui a morbidade da oclusão aguda ${ }^{3,4}$, reduz a taxa de reestenose das lesões de novo ${ }^{5}$ e em pontes de safena ${ }^{6}$, principalmente, quando comparada com os resultados da ATC. Baseado nas investigações de trabalhos randomizados ${ }^{7}$ que mostraram a superioridade do IS sobre a ATC, principalmente nos segmentos proximais das artérias coronárias, seu uso clínico aumentou, significativamente, ficando, ainda assim bastante restrito por duas grandes limitações surgidas nos dias subsequientes ao procedimento: a trombose subaguda e as complicações vasculares com sangramento, decorrentes, em geral, do necessário regime de anticoagulação agressiva ${ }^{8}$.

A nossa experiência com o IS, motivo deste trabalho, iniciou-se em 1994 após a divulgação da proposta do implante ótimo do stent, através da sua impactação na parede arterial coronária com alta pressão no cateter balão. Usando o ultra-som intracoronário (IVUS) ${ }^{9}$ para guiar e controlar esta ótima liberação do stent e sem o conseqüente uso da anticoagulação sistêmica plena, Colombo e col ${ }^{10}$ conseguiram diminuir, de uma só vez, o percentual de complicações vasculares hemorrágicas e a trombose subaguda.

\section{Métodos}

Um grupo de profissionais médicos com semelhante formação hemodinâmica realizou, entre junho/94 a dezembro/96, 2.220 procedimentos terapêuticos invasivos não cirúrgicos na doença arterial coronária (DAC), sendo igualmente analisados, o implante de 783 stents em 660 (29,7\%) pacientes, através da coronariografia convencional. As indicações para o IS (tab. I) foram: 1) lesões de novo ou de indicação eletiva quando o hemodinamicista acreditava em melhor resultado com o stent do que com a ATC: 532 $(67,9 \%)$ pacientes; 2$)$ reestenose coronária: $125(16,0 \%) ; 3)$ resultado subótimo da ATC com ou sem isquemia presente: $64(8,2 \%)$, aqui também incluídos os pacientes com infarto agudo do miocárdio (IAM); 4) oclusão aguda ou provável pós-ATC eletiva: 39 (4,9\%); 5) oclusão total crônica: 23 (3,0\%). Os stents implantados apresentavam diâmetro variando de 3 a $5 \mathrm{~mm}$ e extensão de 9 a 40mm, sendo que 690 $(88,2 \%)$ apresentavam diâmetro de 3 a $3,5 \mathrm{~mm}$ e extensão de 9 a $16 \mathrm{~mm}$. Os principais tipos de stent utilizados (tab. II) foram: Palmaz-Schatz: 320 (40,9\%); Gianturco-Roubin: 228 (29,1\%); NIR: 173(22,0\%); Wallstent: 32(4,1\%); Freedom: 10 $(1,3 \%)$; Cordis: $10(1,3 \%)$ e ACT $10(1,3 \%)$. Todos os pacientes receberam $10000 \mathrm{u}$ de heparina intra-arterial após a inserção da bainha vascular, complementada com mais $2500 \mathrm{u} / \mathrm{h}$ de procedimento e suspensa entre 4 a 24 h após o final. A otimização do IS era considerada alcançada quando o calibre vascular no local do implante permanecia maior que os segmentos proximal e distal à lesão. Com esta intenção, sempre foi utilizada a dilatação e impactação do stent com cateter balão não complacente, no seu calibre nominal ou $0,5 \mathrm{~mm}$ maior do que o diâmetro da artéria coronária e com hiperpressão, variando de 14 a 20 atmosferas (Atm). A seguir, $579(87,8 \%)$ pacientes fizeram uso permanente da associação de ticlopidina $(500 \mathrm{mg} /$ dia $)$ e ácido acetil-salicílico (200mg/dia), via oral, sendo que em $81(12,2 \%)$ realizou-se a anticoagulação plena com a associação de heparina IV (4 dias) e warfarin (2 meses), quando o aspecto angiográfico era considerado não ideal por apresentar irregularidade nos bordos do stent ou aparecimento de pequenas (menor que $5 \mathrm{~mm}$ ) linhas de dissecção junto ao stent. O seguimento clínico foi realizado em $399(60,4 \%)$ pacientes com a utilização da cintilografia miocárdica de esforço e/ou a realização de outra coronariografia.

\section{Resultados}

Dos 660 pacientes, $486(73,6 \%)$ eram homens e 174 $(26,4 \%)$ mulheres com idade variando de 27 a 82 (média $=54 \pm 9$ ) anos. O quadro clínico era compatível com: angina estável em $278(42,1 \%)$ casos, angina instável em 210 $(31,9 \%)$, angina pós-IAM em fase intra-hospitalar em 119 $(18,0 \%)$ e IAM em 53 (8,0\%). A classificação funcional da Sociedade Cardiovascular Canadense (CCS) para insuficiência coronária era: grau 1 em $112(17,0 \%)$ pacientes, grau 2 em $138(20,9 \%)$, grau 3 em $310(47,0 \%)$ e grau 4 em 100 $(15,1 \%)$ (tab. I). As artérias abordadas (tab. II) foram: tronco principal da coronária esquerda (TCE) - 4(0,5\%) stents; descendente anterior (DA) - $400(51,0 \%)$; circunflexa (Cx) - 141 $(18,1 \%)$, sendo $31(4,0 \%)$ na marginal esquerda $(\mathrm{Mg})$, diagonal (DI) - $12(1,5 \%)$; coronária direita (CD) - 190 $(24,2 \%)$, sendo $6(0,8 \%)$ na descendente posterior (DP) e 4 $(0,5 \%)$ na ventricular posterior (VP); ponte de safena (PS) $31(4,1 \%)$ e ponte mamária (PM) - 5(0,6\%). A média de stent por paciente foi de 1,2. Houve sucesso no implante de 770 $(98,0 \%)$ lesões em $646(97,9 \%)$ pacientes e sucesso clínico em 634 (96,0\%) casos (tab. III). Dos 26 (4,0\%) pacientes com

\begin{tabular}{|c|c|c|}
\hline \multicolumn{3}{|c|}{ Tabela I - Dados gerais e indicações para implante de stent } \\
\hline \multicolumn{3}{|l|}{ A - Característica dos pacientes } \\
\hline \multirow[t]{2}{*}{ Número } & $\mathrm{N}$ & $\%$ \\
\hline & 660 & 100,0 \\
\hline \multicolumn{3}{|l|}{ Idade média $=54 \pm 9(27$ a 82$)$} \\
\hline Homem & 486 & 73,6 \\
\hline Mulher & 174 & 26,4 \\
\hline \multicolumn{3}{|l|}{ B - Característica clínica } \\
\hline Angina estável & 278 & 42,1 \\
\hline Angina instável & 210 & 31,9 \\
\hline Angina pós-IAM & 119 & 18,0 \\
\hline IAM & 53 & 8,0 \\
\hline \multicolumn{3}{|c|}{ C - Característica funcional para insuficiência coronária (CCS) } \\
\hline Grau I & 112 & 17,0 \\
\hline Grau II & 138 & 20,9 \\
\hline Grau III & 310 & 47,0 \\
\hline Grau IV & 100 & 15,1 \\
\hline \multicolumn{3}{|l|}{ D - Indicações do implante do stent } \\
\hline Lesão de novo ou eletiva & 532 & 67,9 \\
\hline Reestenose & 125 & 16,0 \\
\hline Resultado sub-ótimo da Atc & 64 & 8,2 \\
\hline Oclusão aguda ou provável pós-ATC & 39 & 4,9 \\
\hline Oclusão crônica & 23 & 3,0 \\
\hline \multicolumn{3}{|l|}{ E - Anticoagulação utilizada } \\
\hline Plena (heparina + warfarin) & 81 & 12,2 \\
\hline Ticlopidina + ácido acetil-salicílico & 579 & 87,8 \\
\hline
\end{tabular}




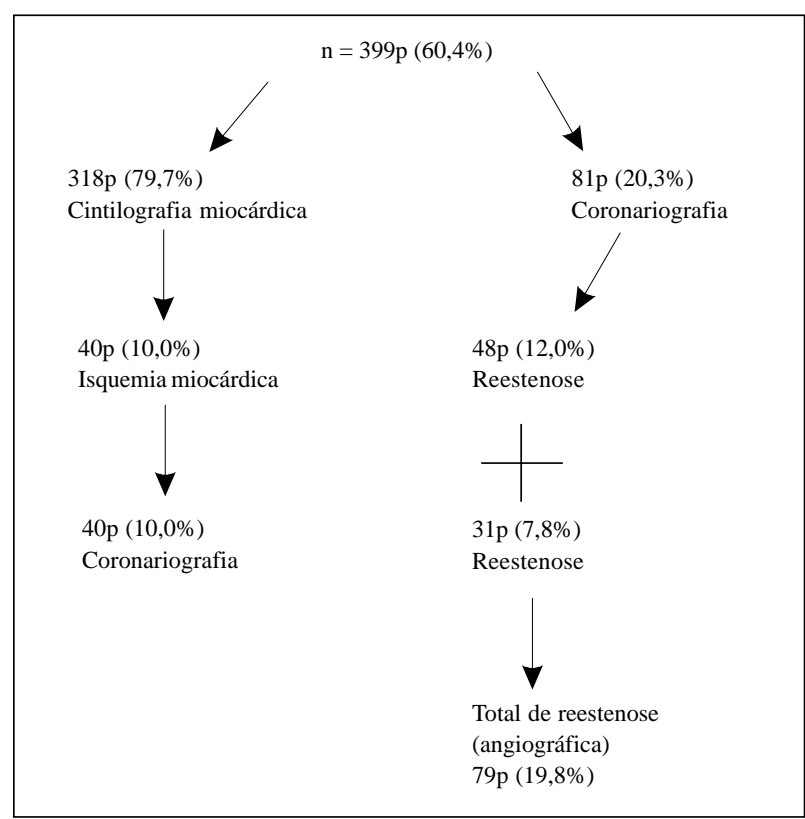

Fig. 1 - Evolução tardia (2 a 12 meses)

Tabela II - Tipos e números de stents e artérias abordadas

A - Tipos e números de stents

Palmaz-Schatz

Gianturco-Roubin

NIR

Wallstent

Freedom

Cordis

ACT

Total de stents

$\mathrm{N} \quad \%$

$320 \quad 40,9$

$228 \quad 29,1$

17322,0

324,1

$10 \quad 1,3$

$10 \quad 1,3$

1,3

B - Vasos abordados com implante de stent TEC

CX

DI

CD

PS

PM

C - Relação stent/paciente: $\quad 1,2$

TEC- tronco de coronária esquerda; DA- artéria descendente anterior; $\mathrm{Cx}$ artéria circunflexa; DI- artéria diagonal; CD- artéria coronária direita; PSponte de safena; PM- ponte mamária. insucesso global imediato, $14(2,1 \%)$ tiveram insucesso no implante e apresentaram a seguinte evolução: IAM em 2 $(0,3 \%)$ pacientes; cirurgia cardíaca em $9(1,4 \%)$ e óbito na sala de hemodinâmica em $3(0,4 \%)$. Os outros $12(1,9 \%)$ tiveram insucesso ainda intra-hospitalar por trombose subaguda com comprovação angiográfica, com a seguinte evolução: IAMem 5 (0,8\%), óbito em 3 $(0,4 \%)$, sem complicação em $4(0,7 \%)$, os quais apresentavam previamente oclusão coronária crônica (tab. IV). A recanalização através da ATC foi realizada com sucesso nos 5 pacientes com IAM póstrombose subaguda. Assim, as complicações maiores no total foram: $22(3,3 \%)$ sendo IAM em $7(1,1 \%)$ casos, cirurgia cardíaca em $9(1,4 \%)$ e óbito em $6(0,8 \%)$. As complicações vasculares (tab. IV) com necessidade de correção cirúrgica e/ou reposição de sangue estiveram presentes em 20 (3,0\%) pacientes, sendo $5(0,8 \%)$ no grupo que utilizou a associação de ticlopidina com aspirina, e 15 (2,2\%) do grupo que fez uso da anticoagulação plena (tab. I). Dos $399(60,4 \%)$ casos, seguidos clinicamente após a alta hospitalar, $318(79,7 \%)$ submeteram-se à cintilografia miocárdica entre $2 \mathrm{a} 12$ meses. O exame foi positivo para isquemia miocárdica na região correspondente à artéria do IS em $40(10,0 \%)$ pacientes que, em seguida submeteram-se a coronariografia que mostrou critério para reestenose em 31 (7,8\%). Os outros $81(20,3 \%)$ foram submetidos a coronariografia sem cintilografia miocárdica prévia. Destes, 48 (12,0\%) pacientes mostraram critérios angiográficos para reestenose. Assim, dos 399 pacientes acompanhados, $121(30,3 \%)$ submeteram-se a outra coronariografia e houve comprovação angiográfica do retorno de lesões, necessitando de nova revascularização (TLR) em 79 (19,8\%) (fig. 1). Estes apresentaram os seguintes eventos tardios: IAM em 6 (1,5\%), cirurgia cardíaca em 9 (2,3\%), óbito em $4(1,0 \%)$, outra ATC ou procedimento similar em $50(12,5 \%)$ e tratamento clínico isolado em $10(2,5 \%)$ (tab.IV).

\begin{tabular}{|lrr|}
\hline \multicolumn{3}{|c|}{ Tabela III - Resultados intra-hospitalares } \\
\hline & $\mathrm{N}$ & $\%$ \\
\hline & 660 & 100,0 \\
Número de pacientes & 646 & 97,9 \\
Sucesso no implante & 12 & 1,9 \\
Trombose sub-aguda & 634 & 96,0 \\
Sucesso clínico & & \\
\hline
\end{tabular}

\begin{tabular}{|c|c|c|c|c|c|c|c|c|}
\hline \multicolumn{9}{|c|}{ Tabela IV - Eventos ocorridos após implante de stents } \\
\hline & \multicolumn{2}{|c|}{ Durante procedimento } & \multicolumn{2}{|c|}{$\mathrm{Na}$ fase hospitalar } & \multicolumn{2}{|c|}{ Tardios ( 2 a 12 meses) } & \multicolumn{2}{|c|}{ Cumulativos (até 12 meses) } \\
\hline & $\mathrm{n}$ & $\%$ & $\mathrm{n}$ & $\%$ & $\mathrm{n}$ & $\%$ & $\mathrm{n}$ & $\%$ \\
\hline Seguimento clínico & 660 & 100 & 660 & 100 & 399 & 60,4 & 399 & 60,4 \\
\hline Infarto do miocárdio & 2 & 0,3 & 5 & 0,8 & 6 & 1,5 & 13 & 3,3 \\
\hline Cirurgia cardíaca & 9 & 1,4 & 0 & 0 & 9 & 2,3 & 18 & 4,5 \\
\hline Óbito & 3 & 0,4 & 3 & 0,4 & 4 & 1,0 & 10 & 2,5 \\
\hline Trombose do stent & 0 & 0 & 12 & 1,9 & 0 & 0 & 12 & 3,0 \\
\hline Outra angioplastia & 0 & 0 & 5 & 0,8 & 50 & 12,5 & 55 & 13,8 \\
\hline Complicação vascular & 0 & 0 & 20 & 3,0 & 0 & 0 & 20 & 5,0 \\
\hline Eventos por paciente & 14 & 2,1 & 45 & 6,9 & 69 & 17,3 & 128 & 32,1 \\
\hline
\end{tabular}




\section{Discussão}

Como o Rio de Janeiro apresenta, como característica própria, vários hospitais de pequeno e médio porte, com um só aparelho para coronariografia em cada hospital e com profissionais hemodinamicistas trabalhando em vários desses hospitais, seria necessário um estudo multicêntrico ${ }^{11}$ para análise, numericamente expressiva, da terapêutica intervencionista coronária não cirúrgica. Diante disto, realizou-se o estudo retrospectivo do IS, a partir de 2.220 procedimentos realizados em sete laboratórios de Hemodinâmica, tendo sido analisados 783 IS em 660 (29,7\%) pacientes, com sucesso no implante de $770(98,0 \%)$ lesões em $646(97,9 \%)$ casos, os quais apresentaram sucesso clínico em 634 $(96,0 \%)$. As complicações maiores durante o procedimento e na fase intra-hospitalar (tab. IV) foram de 1,1\% para IAM, 1,3\% para a realização de cirurgia cardíaca e de $0,9 \%$ para o óbito. Esses resultados superponíveis aos melhores de outros grupos ${ }^{12,13}$ foram alcançados a partir de junho de 1994 , após a divulgação do início da experiência de Colombo e col, finalmente publicada, em $1995^{10}$. Esse trabalho, que mantinha a hipótese de que a trombogenicidade do implante do stent não se devia somente ao seu metal, mas, principalmente, a não liberação ótima do stent, mostrada através do IVUS, poderia ser evitada com a expansão do stent através de alta pressão no cateter balão. Conseqüentemente, a partir daí, não haveria mais a necessidade da anticoagulação agressiva que era feita para se evitar a trombose subaguda, mas que, inevitavelmente, aumentava os sangramentos locais e as complicações vasculares. Com esta simplificação, um número maior de hemodinamicistas, ao qual nos incluímos, animaram-se em iniciar suas experiências com o IS, realizando a ótima impactação na parede da artéria coronária e utilizando, após o procedimento, na grande maioria das vezes, somente a associação de ticlopidina e ácido acetilsalicílico. Assim, na nossa experiência a trombose subaguda ocorreu em somente $12(1,9 \%)$ pacientes e foi responsável por $8(1,2 \%)$ das complicações maiores, enquanto 4 $(0,7 \%)$ que apresentavam previamente oclusão crônica evoluíram sem alterações. As complicações vasculares ocorreram em $20(3,0 \%)$ casos, mas somente em $5(0,8 \%)$ da maioria $(87,8 \%)$ que estava em uso da associação de ticlopidina com aspirina. Assim, a maioria das complicações vasculares $(2,2 \%)$ ocorreu com o uso da anticoagulação plena, que foi utilizada quando o aspecto angiográfico parecia não ser o ideal, o que ocorreu em $81(12,2 \%)$ pacientes mesmo após a impactação do stent com alta pressão no cateter balão. Embora sem o IVUS, como guia e controle do IS, estes dados estão próximos aos encontrados no estudo piloto BENESTENT-II ${ }^{14}$. Nesse, já na sua fase 4 , mostrava, com o implante de um tipo específico de stent (Palmaz-Schatz coberto com heparina) significativa diminuição destas complicações originando, como repercussão, um menor período de internação hospitalar.

Embora na nossa casuística, o perfil clínico tenha mostrado instabilidade clínica em 57,9\% dos casos e a CCS, graus 3 e 4 , estivesse presente em $62,1 \%$ dos pacientes, a maioria dos IS teve alto percentual de sucesso, embora realizados mais em lesões de novo (67,9\%), em geral, após a melhora clínica.

Todas as principais artérias coronárias tiveram sua lesões graves abordadas, sendo a mais freqüente a DA $(51,0 \%)$, onde a indicação preferencial de revascularização permanece sob intensa pesquisa, para a decisão entre a cirurgia cardíaca de ponte de mamária com ou sem circulação extracorpórea, com ou sem esternotomia ${ }^{15}$, e o IS. Enquanto isto, o maior percentual de IS se faz em lesão de novo e na DA, de forma crescente, devido à facilidade na execução, à segurança do procedimento, ao menor desconforto do paciente, ao menor custo e menor tempo de internação hospitalar. Já no outro extremo, o menor percentual de IS no nosso material apareceu nas pontes cirúrgicas (PS e PM), com total de 4,7\%, mas que pareceu ter uma indicação crescente, como opção não cirúrgica, para pacientes que, numa nova cirurgia, teriam maior morbidade e mortalidade.

A nossa segunda indicação mais freqüente do IS foi nas lesões reestenóticas pós-ATC $(16,0 \%)$, constituindo fato tradicionalmente aceito desde a aprovação emprego do stent, já que com o IS, a $2^{\text {a }}$ reestenose tem um percentual menor em relação a ATC ou outros procedimentos. Da mesma forma, embora estudos anteriores tenham sugerido que nas lesões reestenóticas, o benefício tardio pós-IS seja menor que nas lesões de novo, após a otimização do implante, o resultado parece ser igual ${ }^{16}$.

Outra indicação também tradicionalmente aceita é a oclusão aguda ou provável durante a ATC, na qual o IS veio dar mais segurança ao procedimento e tranqüilidade ao hemodinamicista. Nestes casos, o nosso percentual foi de $4,9 \%$ e é semelhante ao que tínhamos há poucos anos, com as insuflações prolongadas com ou sem cateter de perfusão, numa tentativa de se evitar a cirurgia cardíaca de urgência, para corrigir o quadro isquêmico agudo. Com a possibilidade do IS, o percentual de cirurgia cardíaca pós-ATC, principalmente, nas situações eletivas, agora aproxima-se de zero. A nossa média de cirurgia cardíaca pós-IS foi de 1,4\% e de óbito em toda a fase intra-hospitalar de $0,8 \%$, constituindo baixos índices, principalmente, numa população com freqüente alto risco.

Novas fronteiras de indicação do IS estão se abrindo e em fase de julgamento, tais como o seu emprego em pequenos vasos, lesões de bifurcação, lesões difusas, lesões com oclusão total crônica e lesões culpadas por IAM. Nesta última situação, realizamos o IS em 53 (8,0\%) pacientes e há uma tendência de maior emprego do stent, porque, embora a ATC isolada tenha o mesmo percentual de sucesso imediato que o IS, a reoclusão e a reestenose pós-ATC são a maior causa da morbidade e de repetição de procedimento de revascularizaçãotardias ${ }^{17}$. Da mesma forma, na oclusão crônica, que na nossa experiência foi de $3,0 \%$, há uma preferência para se utilizar o IS já que a taxa de reestenose pós-ATC é muito alta (68\%) enquanto que pós-stent está em torno de $40 \%{ }^{18}$.

Neste campo do IS, de conhecimentos evolutivos rápidos, em que as verdades de hoje sofrem modificações a curto prazo, sabe-se que a otimização do IS depende, funda- 
mentalmente, da seleção da lesão a ser abordada, da seleção do tipo de stent a ser utilizado e como realizá-lo. Para o IS utilizamos diversos tipos de stent, mais freqüentemente, o Palmaz-Schatz em 40,9\%, o de Gianturco-Roubin em 29, $1 \%$ e o stent NIR em 20,2\%, nas mais diversas situações anatômicas e mesmo em pacientes com lesões multiarteriais, embora na nossa experiência a relação stent/paciente de 1,2 indique o emprego mais freqüente em lesão uniarterial. Dentre os stents mais utilizados, selecionamos para o implante o tipo tubular (Palmaz-Schatz) que foi empregado, preferentemente, em lesões com grande placa aterosclerótica, lesões reestenóticas, lesões-aorto-ostiais, lesões com calcificação, lesões totais crônicas e lesões em ponte de safena. Já o stent espiralado monofilamentar(Gianturco-Roubin) foi, preferentemente, implantado nas lesões em curva, lesões que envolviam ramos laterais, lesões com dissecções e lesões situadas em vasos tortuosos, aproveitando a sua característica de maior flexibilidade.

É sabido que o stent é um instrumento mecânico que realiza o seu principal benefício, aumentando a luz do vaso. E isto se faz quando o stent cobre a maior quantidade possível de placa obstrutiva mantendo de imediato com sua força radial a perviabilidade do vaso sem trombose e, a seguir, sem reestenose tardia. Quanto à reestenose, temos adquirido muitos conhecimentos nesta época pós-ATC e procedimentos similares. Assim, sabe-se que com o IS o ganho inicial do calibre do vaso é maior e a perda tardia é maior ou igual a ATC o que leva a um ganho total maior e, daí, a uma provável menor reestenose clínica, mesmo que a reestenose anatômica seja mais intensa ${ }^{19}$.

A revolucionária técnica de Colombo e col ${ }^{10}$ para o IS parece também estar mostrando, através de diversos ensaios clínicos ${ }^{20,21}$, uma significativa menor taxa de reestenose, quando comparada com a ATC. Trabalhos isolados não randomizados também têm mostrado uma taxa média de reestenose entre $12 \%$ e $27 \%{ }^{19}$ mas em situações especiais pode ser mais alta. Assim, quando há o emprego de stents múltiplos é de $49 \%$ em lesões de vaso menor que $3 \mathrm{~mm}$ de diâmetro, é de $48 \%$, e quando se utiliza pressão de impactação menor que $12 \mathrm{~atm}$ é de $43 \%{ }^{22}$.

No nosso seguimento tardio de $399(60,4 \%)$ pacientes realizou-se outra coronariografia em 121 (30,3\%) casos, onde houve a comprovação angiográfica de lesões, intrastent, que necessitariam de nova revascularização (TLR) em
79(19,8\%). Esta taxa (fig. 1), embora dentro da faixa obtida por outros autores, ainda permanece significativa e maior do que a encontrada, quando se utilizou o IVUS ${ }^{23}$. Este, em geral, orientou a análise do tipo de lesão a se abordar, o tipo e o tamanho do stent a se utilizar, a necessidade de outro tipo de procedimento com a verificação de certeza em relação a ótima impactação do stent.

Utilizando somente a coronariografia, sem angiografia quantitativa e acreditando ter atingido o implante ótimo do stent, obtivemos, igualmente a outros autores ${ }^{24}$, ótimos resultados imediatos. Assim há, sem dúvida, uso crescente do stent, de tal forma que houve a utilização do IS em 29,7\% do total de procedimentos realizados, e nos últimos 100 procedimentos, acima de $60 \%$ deles incluem o implante de pelo menos um stent. Mas os nossos resultados tardios, embora melhores do que os habitualmente encontrado com a ATC, ainda não são ideais. A evolução tardia dos pacientes mostrou que 4,8\% deles apresentaram complicações maiores, $12,5 \%$ submeteram-se a outra ATC ou procedimento similare 2,5\% permaneceram em tratamentoclínico. Assim, os eventos cumulativos não desejáveis, em 12 meses, foram de $32,1 \%$ e destes, mais da metade em decorrência da reestenose (tab. IV).

Como diminuir a taxa de reestenose após o IS? Além do possível papel complementar de drogas, como o bloqueador do receptor plaquetário IIb/IIIa e outras, da terapia através da biologia molecular, da terapia com radiação vascular ${ }^{25}$ durante e após o IS, do emprego do stent heparinizado ou irradiado, ou recoberto com veia, seria a utilização do IVUS imprescindível para a impactação ótima do stent e daí se alcançar menor taxa de reestenose? Algumas perguntas sobre a reestenose já começam a ser respondidas e o estudo piloto STRESS-III ${ }^{26}$ mostrou que a utilização do IVUS durante o IS alterou o plano terapêutico em $56 \%$ dos implantes, exigindo maior pressão do balão em $41 \%$, o emprego do cateter balão com maior calibre em $44 \%$ e o emprego de um stent adicional em $21 \%$, sem no entanto, melhorar o resultado imediato ou diminuir as complicações, devendo-se aguardar ainda a evolução tardia para se saber qual o impacto do IVUS sobre os resultados finais.

De qualquer forma, o IS se mostrou até hoje um indiscutível avanço tecnológico que permitiu ao hemodinamicista executar o tratamento da lesão obstrutiva na DAC com mais segurança, alto índice de sucesso imediato, poucas complicações e menor taxa de reestenose do que a ATC. 


\section{Referências}

1. Dotter TC - Transluminally placed coilspring endarterial tube grafts: longterm patency in canine popliteal artery. Invest Radiol 1969; 4: 329-32.

2. Sigwart V, Puel J, Mirkovitch V, Jeffer F, Kappenberger L - Intravascular stents to prevent occlusion and restenosis transluminal angioplasty. N Engl J Med 1987; 316: 701-6.

3. George BS, Voorhees WD, Roubin GS et al - Multicenter investigation of coronary stenting to treat acute or threatened closure after percutaneous transluminal coronary angioplasty: clinical and angiographic outcomes. J Am Coll Cardiol 1993; 22: 135-43

4. Roubin GS, Cannon AD, Agrawal SK et al - Intracoronary stenting for acute or threatened closure complicating percutaneous transluminal coronary angioplasty . Circulation 1992; 85: 916-27.

5. Schatz RA, Goldberg S, Leon MB et al - Clinical experience with the PalmazSchatz coronary stent. J Am Coll Cardiol 1991; 17: 155B-9B.

6. Platko WP, Hollman J, Whitlow PL, Franco I - Percutaneous translumina angioplasty of saphenous vein graft stenosis: long term follow-up. J Am Coll Cardiol 1989; 14: 1645-50.

7. Fischman DL, Leon MB, Baim D et al - A randomized comparison of coronary stent placement and balloon angioplasty in the treatment of coronary artery disease. N Engl J Med 1994; 331: 496-501.

8. Nath FC, Muller DWM, Ellis SG et al - Thrombosis of a flexible coil coronary stent: frequency, predictors, and clinical outcomes. J Am Coll Cardiol 1993; 21 : 622-7.

9. Nakamura S, Colombo A, Gaglione S et al - Intracoronary ultrasound observations during stent implantation. Circulation 1994; 89: 2026-34.

10. Colombo A, Hall P, Nakamura S et al - Intracoronary stenting without anticoagulation accomplished with intravascular ultrasound guidance. Circulation 1995; 91: 1676-88.

11. Romão N, Rodrigues C, Pessanha A et al - Estudo multicêntrico do implante de stent intracoronário na cidade do Rio de Janeiro. Arq Bras Cardiol 1996; 67 (supl. I) 113 .

12. Versaci F, Gaspardone A, Tomai F, Crea F, ChiarielloL, Gioffre PA - A comparison of coronary-artery stenting with angioplasty for isolated stenosis of the proximal left anterior descending coronary artery. N Engl J Med 1997; 336: 817-22.

13. Stone GW, Brodie B, Griffin J et al - A prospective, multicenter trial of primary stenting in acute myocardial infarction - the PAMI stent pilot study. Circulation 1996; 94(supl I): 570

14. Serruys P, Emanuelsson H, Vander Giessen W et al - Heparin-coated Palmaz-
Schatz stent in human coronary arteries: early outcome of the BENESTENT-II pilot study. Circulation 1996; 93: 412-22.

15. Calafiore AM, Giammarco G, Teodori Get al-Left anterior descending coronary artery grafting via left anterior small thoracotomy without cardiopulmonary bypass. Ann Thorac Surg 1996; 61: 1658-65.

16. Hong MK, Kent KM, Sattler LF et al - Are long-term results different when stents are used in de novo versus restenotic lesions? Circulation 1996; 94(suppl I): I331.

17. Mattos LA, Feres F, Abizaid A et al - Repeat revascularization procedures after primary PTCA for myocardial infarction 1996; 94(suppl I): I330.

18. Nienaber CA, Fratz S, Lund GK, Stiel GM - Primary stent placement or balloon angioplasty for chronic coronary occlusions: a matched pair analysis in $100 \mathrm{pa}-$ tients. Circulation 1996; 94(suppl I): I 686

19. Serra A, Masotti M, Aviles FF et al - Stent vs angioplasty restenosis trial (START). Influence of vessel size on angiographic restenosis. Circulation 1996; 94(suppl I): I92.

20. Erbel R, Hande M, Hopp HW, Meyer J, Swars H - Restenosis stent (REST) study: randomized trial comparing and balloon angioplasty for treatment of restenosis after balloon angioplasty. J Am Coll Cardiol 1996; 27(suppl A): 139A.

21. Wong SC, Baim DS, Schatz R-A et al - Immediate results and late outcomes after stent implantation in saphenous vein graft lesion: the multicenter V. S. PalmazSchatz stent experience. J Am Coll Cardiol 1995; 26: 704-12.

22. Lablanche JM, Danchin N, Grollier Get al - Factors predictive of restenosis after stent implantation managed by ticlopidine and aspirin. Circulation 1996; 94(suppl I): I-256.

23. Goldberg SL, Colombo A, Nakamura S, Almagor Y, Maiello L, Tobis JM - The benefit of intracoronary ultrasound in the deployment of Palmaz-Schatz stents. J Am Coll Cardiol 1994; 24: 996-1003.

24. Goods CM, Mathur A, Liu MW et al - Intracoronary stenting using slotted tubular stents without intravascular ultrasound and anticoagulation. Cather Cardiovasc Diag 1996; 39: 341-5

25. Teirstein PS, Massullo V, Jani S et al - Radiation therapy following coronary stenting 6 month follow-up of a randomized clinical trial. Circulation 1996; 94(suppl I): I-210.

26. Strain JE, Rehman DE, Fischman D, Cohen N, Moses JW-STRESS III; preliminary acute results of IVUS vs non-IVUS stenting. Circulation 1996; 94(suppl I): I-200. 